\title{
Changes to the mtDNA copy number during yeast culture growth
}

Ben Galeota-Sprung ${ }^{1,2}$, Amy Fernandez ${ }^{1}$, Paul Sniegowski ${ }^{1}$

${ }^{1}$ University of Pennsylvania

${ }^{2}$ Corresponding author 


\section{Abstract}

The yeast mitochondrial network is dynamic, with frequent fusion and fission events among mitochondria. Notable changes in mitochondrial morphology and function accompany changes in physiological state. Differences in mtDNA copy number among strains and growth conditions have been observed, but it has not been previously shown that the mtDNA copy number changes substantially over time in a clonal growing culture. Here we show that there are fairly dramatic $(3 \mathrm{x}-5 \mathrm{x})$ changes to the mtDNA copy number per cell in growing cultures of Saccharomyces cerevisiae, on a timescale of hours to days. While these changes appear to be connected to the shift from fermentation to respiration, it is possible that they are more simply associated with changes in growth rate. Our work suggests that the mtDNA copy number is a dynamic phenotype, a finding that has important implications for studies of the regulation of mtDNA copy number. 


\section{Introduction}

Mitochondria are membrane-enclosed organelles that originate from an ancient symbiosis (Gray 2017; Sagan 1967). Mitochondria enable a variety of capabilities, the most important of which is the production of ATP via aerobic respiration. To a varying degree across the eukaryotic tree of life, most originally mitochondrial genes have been assimilated into the nuclear genome (Malina, Larsson, and Nielsen 2018), but in nearly all cases an independent mitochondrial genome (mtDNA) is maintained within the mitochondria. Generally there are multiple copies of mtDNA per individual cell. For example, in humans, there are hundreds to thousands to tens of thousands of mtDNA copies per cell with considerable variation between tissue type (D'Erchia et al. 2015; Dimmock et al. 2010; Miller et al. 2003; Wai et al. 2010) as well as substantial inter-individual variation (Kaaman et al. 2007) in copy number. In humans, deficiency in mtDNA copy number is associated with a variety of human disease phenotypes (Elpeleg, Mandel, and Saada 2002; Kornblum et al. 2013; Pyle et al. 2016; Zeviani and Di Donato 2004).

The mtDNA copy number in the yeast Saccharomyces cerevisiae is thought to vary somewhat by strain (Miyakawa 2017). An early study (Hall, Nagley, and Linnane 1976) found that 16-25\% of all cellular DNA is mitochondrial; given an mtDNA genome of 75-85 kbp and a nuclear genome of $\sim 12 \mathrm{Mbp}$, this suggests a copy number of $\sim 25$ to $\sim 50$ per haploid genome, a range generally borne out by subsequent studies (Lebedeva and Shadel 2007; Solieri 2010; Donald H. Williamson 2002), though copy numbers as high as 200 have been observed in diploids (Miyakawa et al. 2004). In yeast, as in other organisms, mtDNA is packed into protein-DNA complexes call nucleoids (Miyakawa 2017; Westermann 2014; Donald H. Williamson 2002; Donald H. Williamson and Slonimski 1976). These contain mostly linear mtDNA in polydisperse (i.e. not discrete) linear tandem arrays containing on average one to two copies of the mitochondrial genome (Freel, Friedrich, and Schacherer 2015; Maleszka, Skelly, and ClarkWalker 1991), though under anaerobic conditions there may be many fewer nucleoids with many more mtDNA per nucleoid (Chen and Butow 2005). Two recent studies have found 10-20 mtDNA copies per haploid cell, and up to roughly threefold variability introduced by various gene deletions (Göke et al. 2019; Puddu et al. 2019).

The mitochondria within the yeast cell form a highly dynamic network: fusion and fission events occur very frequently (Jakobs et al. 2003; Simon, Karmon, and Pon 1997). There is generally a single "giant" mitochondrion that is much bigger than the others, and the number of spatially distinct mitochondria varies with physiological state, being lowest in rapidly growing cells and highest in stationary phase cells (Stevens 1981). S. cerevisiae that are grown with glucose as a carbon source characteristically ferment it into ethanol and then transition into a fully respiring physiology (the diauxic shift) upon the exhaustion of glucose supplies. The mitochondrial network undergoes substantial changes during this transition: the total mitochondrial volume as a proportion of cell volume increases greatly (Stevens, 1981; Bartolomeo et al., 2020) and the mitochondrial role transitions from biosynthetic hub to energy generator, reflected in a dramatic shift in the mitochondrial proteome, as well as a general increase in mitochondrial protein synthesis (Bartolomeo et al. 2020).

In this study, we investigate changes in mtDNA copy number over time by sampling repeatedly from growing cultures of $S$. cerevisiae. We interrogate mtDNA copy number change on the scale of hours and days in both a common laboratory strain and a natural isolate, using both qPCR and WGS 
approaches. We find large (3x-5x) copy number increases as cultures shift from a rapidly growing physiological state to a stationary number of cells, with substantial differences between strains as well as effects of ploidy. We also confirm the reverse phenomenon-that when slowly growing cells are transferred to fresh media, the mtDNA copy number drops. These results re-contextualize both longstanding observations of changes in mitochondrial volume and morphology as well as more recent findings of changes to mitochondrial protein production as the growth mode changes, and suggest that the mtDNA copy number is a dynamic and plastic phenotype. 


\section{Results}

\section{The mtDNA copy number increases $\sim 4$-fold over time in growing cultures}

We inoculated replicate cultures by dilution into rich liquid media containing a fermentable carbon source (YPD), or nonfermentable carbon sources (YPG, YPEG), and tracked the relative ratio of mtDNA to genomic DNA (mt/gDNA ratio) over time by qPCR (see Methods). We used both a standard laboratory background (W303) as well as a natural isolate. In haploid and diploid laboratory strains, the $\mathrm{mt} / \mathrm{gDNA}$ ratio increased approximately 4 - to 5-fold over the course of $\sim 4$ days (Fig. 1a, gray). The relative increase in a haploid derived from a wild isolate was somewhat less ( 3.5 -fold) while a wild diploid strain displayed an initial increase in mt/gDNA ratio followed by a subsequent fall. This wild diploid strain was observed to be a ready sporulator, likely explaining this particular phenomenon (see Discussion).

A Haploids in YPD

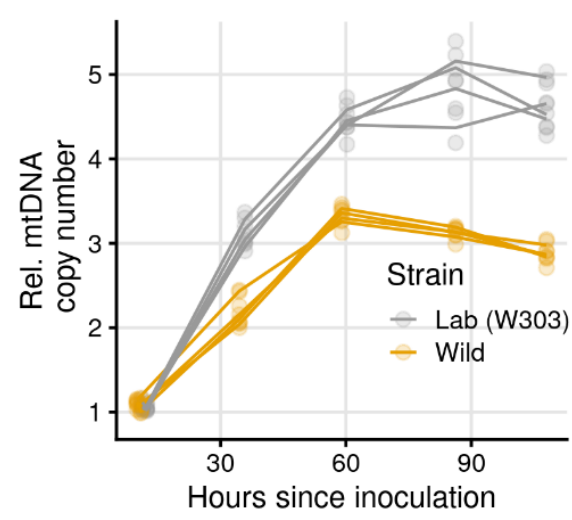

B Diploids in YPD

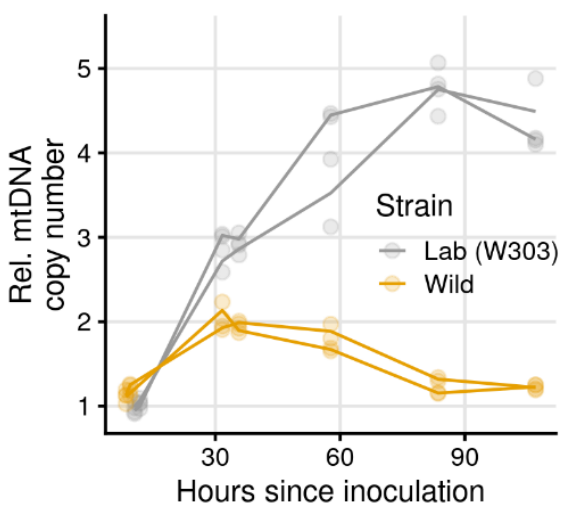

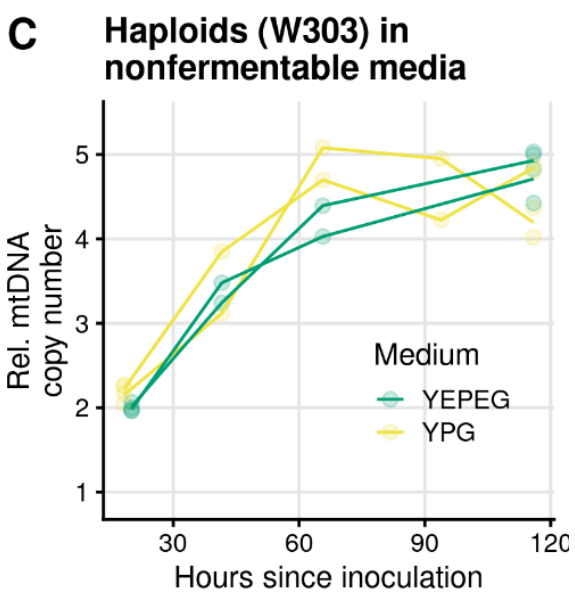

Fig. 1 mtDNA copy number over time of replicate cultures inoculated into $50 \mathrm{~mL}$ YPD of (A) haploid and (B) diploid lab and wild strains, or (C) haploid lab strains inoculated into $50 \mathrm{~mL}$ nonfermentable media. Inoculations were 1/2000 dilutions from saturated cultures. Two replicate qPCR measurements (circles) were performed for each extraction at each time point; the lines track the mean of the two measurements. The first extraction was always performed at $\mathrm{A}_{600}=0.5$ which corresponds to $\sim 3 \mathrm{e} 07$ cells $/ \mathrm{mL}$ in haploid strains and $\sim 2.4 \mathrm{e} 07$ cells $/ \mathrm{mL}$ in diploid strains. Four subsequent extractions were carried out for each culture at approximately 24-hour intervals. qPCR measurements of relative mtDNA copy number were performed via the relative standard curve method (see Methods).

\section{The timing of the mtDNA copy number increase is associated with a decrease in growth rate}

In order to more carefully study the phenomenon of rising mtDNA copy number in a growing culture, we performed a denser set of DNA extractions (17 in total) over a similar period of time, along with corresponding density measurements, from a single growing culture. Characteristically, when $S$. cerevisiae grown in rich media exhaust the supply of glucose, the culture enters the diauxic shift, transitioning from fermentation of glucose to oxidative respiration of ethanol. The rate of growth slows considerably at this point. In this experiment, we observed (Fig. 2a) a roughly 4-fold increase in mtDNA copy number, similar to the results from the experiment displayed in Fig 1a. Inspection of the 
first $\sim 20$ hours of growth (Fig 2b; note the change in slopes) reveals that the slowing of growth rate characteristic of the diauxic shift occurs at about $\sim 14 \mathrm{~h}$ after inoculation in our growth conditions; this is also when the mtDNA copy number begins to increase. Thus the onset of the increase in mtDNA copy number is temporally associated with the diauxic shift during growth in glucose-containing media.
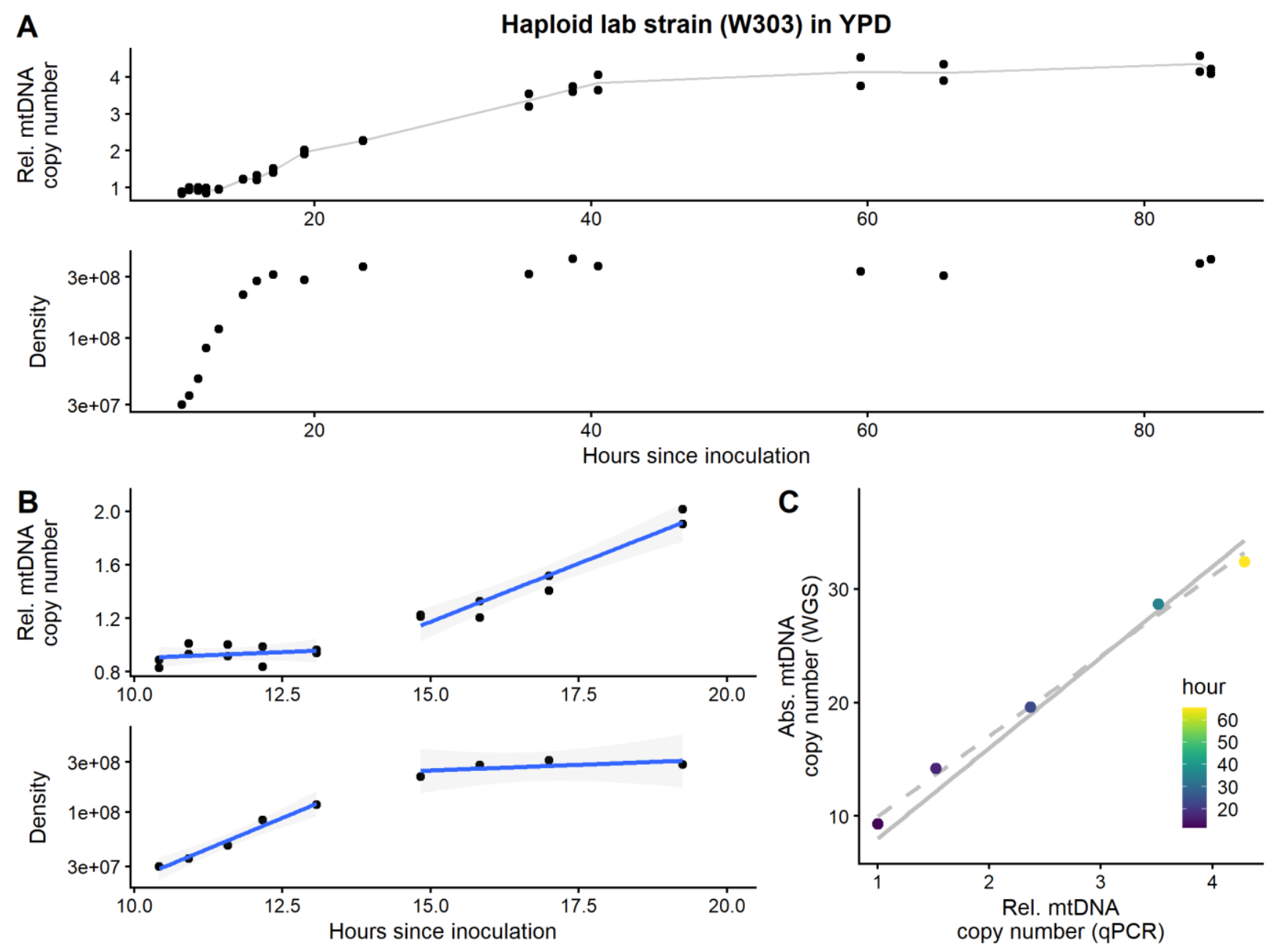

Fig. 2 (A) As in the experiment shown in Fig. 1, the mt/gDNA ratio rises over time in a culture of the haploid lab strain. (B) Detail of first 20 hours. The onset of the rise is associated with the slowing of growth as fermentation ends; blue lines are linear regressions of copy number, top, and cell density, bottom. (C) qPCR estimates of the mt/gDNA ratio are wellreproduced by whole genome sequencing (see Methods) although the fold change obtained is a bit less, $\sim 3.5 \mathrm{x}$ by sequencing vs $\sim 4.3 \mathrm{x}$ by qPCR. The difference is shown by the dotted regression line compared to the solid line which represents a perfectly matched relative increase between the two assays.

As a control to rule out any effect of total time in culture, we checked that the relative mtDNA copy number would remain at $\sim 1$ if a culture was repeatedly transferred while still in fermentation. This indeed was the case: a culture that had been transferred four times consecutively while in fermentation (total growth time of $\sim 60$ hours) maintained a relative mtDNA copy number of $\sim 1$ (Fig S5). 


\section{The minimum number of mtDNA copies in haploids is about 9 and increases to over $\mathbf{3 0}$}

Our primary assay for investigating copy number change was a qPCR assay that quantified the $\mathrm{mt} / \mathrm{gDNA}$ ratio. We also used read depth from WGS in order to both verify our qPCR results with an independent assay and to estimate absolute mtDNA copy number. For 5 of the 17 timepoints for which qPCR assays were performed, a WGS-based assay to determine mtDNA copy number was also carried out (see Methods). Fig 2c displays the results. The two assays correspond quite well although the fold increase is slightly attenuated in the WGS assay compared to the qPCR assay. We found that mtDNA copy number ranges from $\sim 9$ during fermentation to $>30$ in respiration and/or stationary phase, in haploids. Because our qPCR results indicate (Figs $1 \mathrm{a}$ and $1 \mathrm{~b}$ ) very similar relative $\mathrm{mt} / \mathrm{gDNA}$ results in the haploid and diploid lab strains, these estimates of mtDNA copy number per cell should be doubled for those diploids. As controls for the WGS assay, we assayed the copy number of rRNA repeats, the CUP1 locus, and Ty transposons, which did not change over time (Fig. S1).

\section{An mtDNA copy number increase also occurs under non-fermentative conditions}

We also found that a substantial increase in mtDNA copy number occurs when the lab strain is grown on nonfermentable carbon sources - glycerol or ethanol+glycerol. The final copy number obtained at $100+$ hours after inoculation is very similar to that obtained during growth on glucose (compare Fig 3 to Fig 1a), approximately 4- to 5-fold the copy number during rapid fermentative growth. However the first measurement of mtDNA copy number-measured in all conditions at $\mathrm{A}_{600}=0.5$-is about twice as high as during fermentative growth.

\section{The mtDNA copy number drops rapidly upon transfer from stationary phase}

To form a fuller picture of mtDNA copy number dynamics, we investigated the time scale of the decline in mtDNA copy number that must by implication occur after dilutive transfer from a saturated culture to fresh media. To do so, we transferred a relatively large number of stationary cells (sufficient that extractions could be immediately performed) to fresh YPD and began regular extractions at once. The results of this experiment are displayed in Fig 4. The expected drop occurred rapidly at first, mostly taking place between 1 and 6 hours after transfer, or about 3-4 generations. These observations are broadly consistent with those obtained by (D. H. Williamson and Moustacchi 1971). 


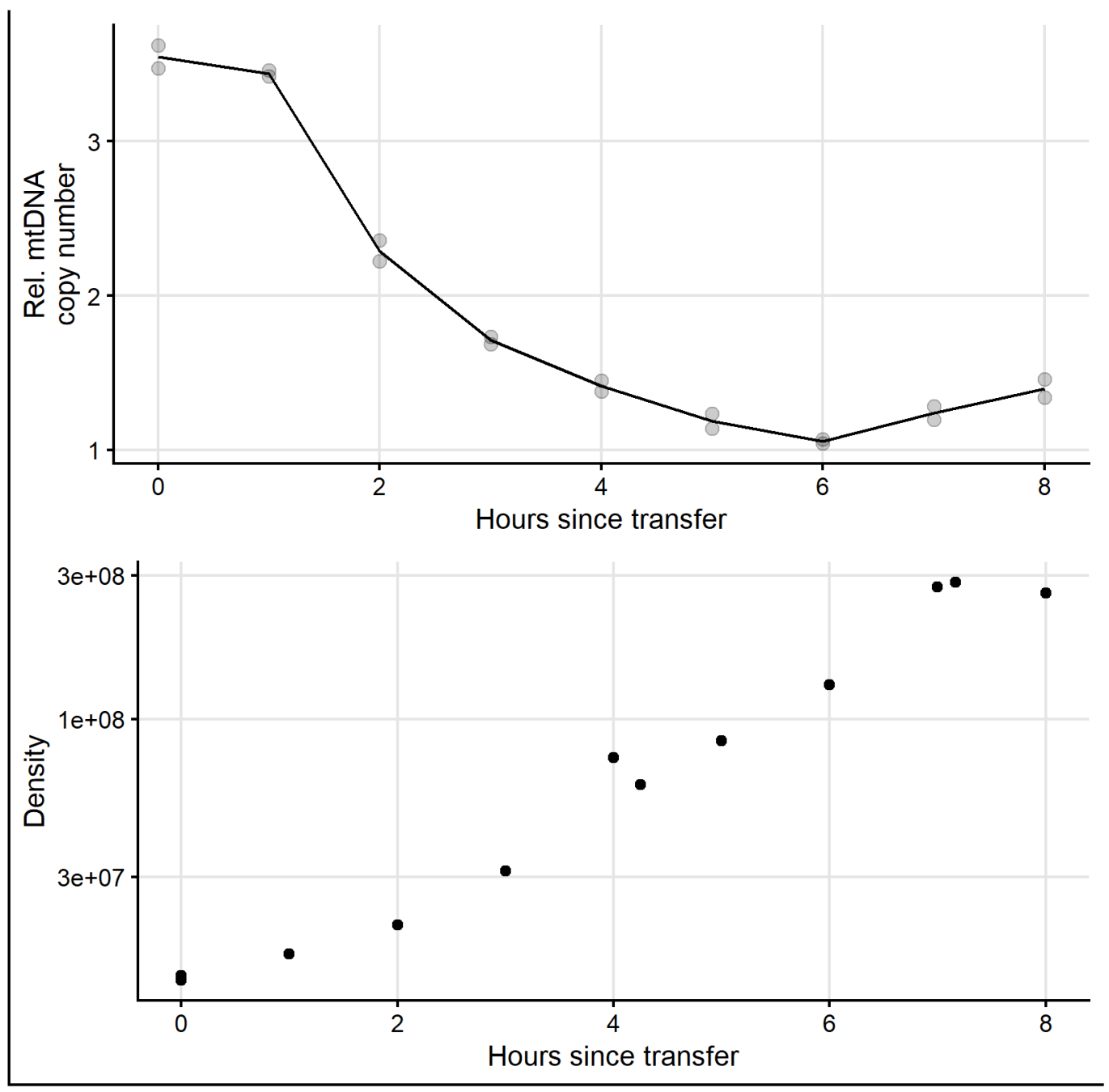

Fig. 3 A saturated culture of the haploid lab strain was transferred, diluting 1/30, to fresh YPD. The relatively mild dilution (1/2000 dilutions were performed in all other experiments) permitted DNA extractions of small portions of the culture to begin immediately after transfer. Extractions for subsequent qPCR assays and density measurements were performed at 1hour intervals for 8 hours.

\section{Discussion}

It has long been recognized that the mitochondrial network in yeast is highly dynamic, and undergoes substantial physiological changes during growth, especially (for Crabtree-positive yeasts) during the shift from fermentation to respiration when grown on a fermentable carbon source such as glucose. During this physiological shift, the appearance of the mitochondrial network changes, as does the total mitochondrial volume (both proportional and absolute), the total rate of production of mitochondrial proteins, and the composition of the mitochondrial proteome. Here we show that, during these changes, the number of copies of the mitochondrial genome per nuclear genome does not remain fairly constant, but instead varies by $\sim 4$-fold (in a lab strain, somewhat less in a wild strain), with the copy number lowest during rapid fermentative growth and increasing thereafter. The magnitude of this change is 
surprising. For example, a previous study found a $\sim 30 \%$ difference in mtDNA copy number between aerobic and anaerobic conditions (Miyakawa et al. 2004).

It is tempting to posit that because the capacities of mitochondria are most crucial during respiration, production of mitochondria is deprioritized during fermentation, accounting for the phenomenon described here, and that production of more mitochondrial genomes is deferred until they are most needed. Indeed the data bear this out in the sense that when grown under nonfermentative conditions (i.e. with glycerol or glycerol and ethanol as the carbon source) the mtDNA copy number is not as low as during growth in glucose (compare Fig. 1c to Figs. 1a and 1b), at least at similar densities. However, the mtDNA copy number also increases fairly sharply over time in nonfermentative conditions (Fig. 1c). Thus the diauxic shift from one physiological state to another is at best an incomplete explanation for the phenomena observed here.

In S. cerevisiae, mtDNA replication is not linked to the cell cycle (Miyakawa et al., 2004; Williamson $\&$ Moustacchi, 1971). Therefore, the rate of cellular or genomic growth (i.e. the rate of budding) is not necessarily coupled to the rate of mtDNA production in the short term. A toy model that can recapitulate both the rise and fall in mtDNA copy number observed here is that changes in mitochondrial growth rate always lag behind changes in cellular (=genomic) growth rate. Under this model, when cells are transferred from conditions of slow growth or stationary phase to fresh media, the cellular growth rate initially increases more rapidly than the mitochondrial growth rate, which causes the mtDNA copy number to fall. The mitochondrial growth subsequently catches up, which stabilizes the mtDNA copy number. When the cellular growth rate begins to slow as resources become exhausted, the mitochondrial growth rate does not immediately slow, causing the mtDNA copy number to increase. Such a model is illustrated in Fig. 4. Under this framework, the increases (Figs. 1, 2) and drops (Fig. 3) in mtDNA copy number that we observed are the rising and falling aspects of the full cycle that would occur with repeated serial transfers. Why such a lag should occur is not clear. One potential adaptive explanation is connected to the two modes of reproduction in yeast. Asexual reproduction (budding) is associated with rapid growth, while sexual reproduction (sporulation) tends to occur when environmental conditions become challenging and growth ceases. During sporulation, mitochondria are partitioned into four offspring, but only a little more than half of the total mtDNA makes it into one of the four ascospores (Brewer and Fangman 1980). Thus a sporulating diploid requires a high mtDNA copy number in order for each of its four haploid offspring to possess an adequate number of mtDNA copies. 

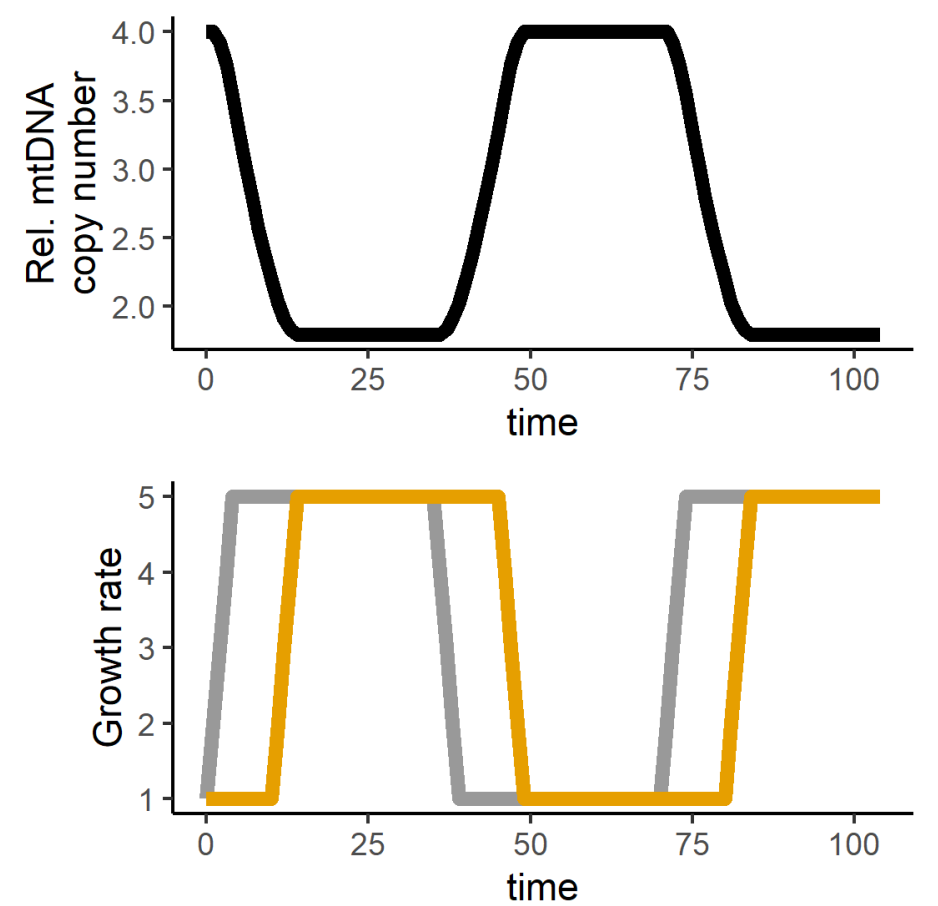

Type of growth

gDNA

mtDNA

Fig. 4 A simulation of a simple model that can produce patterns similar to those observed. Both cellular (i.e. gDNA) and mitochondrial growth rates alternate between the same two values, but changes to the mtDNA growth rate lag behind changes to the gDNA growth rate. This causes an increase in the relative mtDNA copy number when the cellular growth rate slows, and a decrease in mtDNA copy number when the cellular growth rate increases.

It appears that sporulation itself has a significant effect on mtDNA copy number relative to genomic copy number: our wild diploid strain, which is a very ready sporulator, displayed very different mtDNA copy number dynamics (Fig 1b) than the lab diploid strain, which must be induced to sporulate. We speculate that this is because the single genome doubling that occurs in meiosis is not accompanied by a corresponding increase in mtDNA copy number (Brewer and Fangman 1980); thus the mt/gDNA ratio falls by a factor of $\sim 2$ as a result of meiosis.

Our findings show that mtDNA copy number is a dynamic phenotype in which the average copy number per cell depends on physiological state and culture time. This suggests that differences in mtDNA copy number between strains should be investigated carefully, ideally with time-series data, to ensure that apples-to-apples comparisons are made, especially between strains with difference growth rates. One limitation of our work is that we have measured the mean copy number per cell as a bulk property of the culture. Future work might examine the distribution of mtDNA copy number per individual cell over time in order to more fully elucidate the manner in which these changes occur. 


\section{References}

Bartolomeo, Francesca Di et al. 2020. “Absolute Yeast Mitochondrial Proteome Quantification Reveals Trade-off between Biosynthesis and Energy Generation during Diauxic Shift." Proceedings of the National Academy of Sciences 117(13): 7524-35.

Brewer, B. J., and W. L. Fangman. 1980. "Preferential Inclusion of Extrachromosomal Genetic Elements in Yeast Meiotic Spores." Proceedings of the National Academy of Sciences 77(9): 5380-84.

Chen, Xin Jie, and Ronald A. Butow. 2005. "The Organization and Inheritance of the Mitochondrial Genome." Nature Reviews Genetics 6(11): 815-25.

D’Erchia, Anna Maria et al. 2015. “Tissue-Specific MtDNA Abundance from Exome Data and Its Correlation with Mitochondrial Transcription, Mass and Respiratory Activity." Mitochondrion 20: $13-21$.

Dimmock, David, Lin-Ya Tang, Eric S Schmitt, and Lee-Jun C Wong. 2010. “Quantitative Evaluation of the Mitochondrial DNA Depletion Syndrome." Clinical Chemistry 56(7): 1119-27.

Elpeleg, Orly, Hanna Mandel, and Ann Saada. 2002. "Depletion of the Other Genome-Mitochondrial DNA Depletion Syndromes in Humans.” Journal of Molecular Medicine 80(7): 389-96.

Freel, Kelle C., Anne Friedrich, and Joseph Schacherer. 2015. "Mitochondrial Genome Evolution in Yeasts: An All-Encompassing View." FEMS Yeast Research 15(fov023). https://doi.org/10.1093/femsyr/fov023 (May 12, 2021).

Göke, Aylin et al. 2019. "Mrx6 Regulates Mitochondrial DNA Copy Number in Saccharomyces Cerevisiae by Engaging the Evolutionarily Conserved Lon Protease Pim1." Molecular Biology of the Cell 31(7): 527-45.

Gray, Michael W. 2017. "Lynn Margulis and the Endosymbiont Hypothesis: 50 Years Later.” Molecular Biology of the Cell 28(10): 1285-87.

Hall, R. M., P. Nagley, and A. W. Linnane. 1976. "Biogenesis of Mitochondria. XLII. Genetic Analysis of the Control of Cellular Mitochondrial DNA Levels in Saccharomyces Cerevisiae." Molecular \& general genetics: $M G G$ 145(2): 169-75.

Jakobs, Stefan et al. 2003. "Spatial and Temporal Dynamics of Budding Yeast Mitochondria Lacking the Division Component Fis1p.” Journal of Cell Science 116(10): 2005-14.

Kaaman, M. et al. 2007. "Strong Association between Mitochondrial DNA Copy Number and Lipogenesis in Human White Adipose Tissue." Diabetologia 50(12): 2526-33.

Kornblum, Cornelia et al. 2013. "Loss-of-Function Mutations in MGME1 Impair MtDNA Replication and Cause Multisystemic Mitochondrial Disease.” Nature Genetics 45(2): 214-19.

Lebedeva, Maria A., and Gerald S. Shadel. 2007. "Cell Cycle- and Ribonucleotide Reductase-Driven Changes in MtDNA Copy Number Influence MtDNA Inheritance Without Compromising Mitochondrial Gene Expression." Cell Cycle 6(16): 2048-57. 
Maleszka, R, P J Skelly, and G D Clark-Walker. 1991. "Rolling Circle Replication of DNA in Yeast Mitochondria." The EMBO Journal 10(12): 3923-29.

Malina, Carl, Christer Larsson, and Jens Nielsen. 2018. "Yeast Mitochondria: An Overview of Mitochondrial Biology and the Potential of Mitochondrial Systems Biology." FEMS Yeast Research 18(foy040). https://doi.org/10.1093/femsyr/foy040 (May 11, 2021).

Miller, Francis J. et al. 2003. "Precise Determination of Mitochondrial DNA Copy Number in Human Skeletal and Cardiac Muscle by a PCR-based Assay: Lack of Change of Copy Number with Age." Nucleic Acids Research 31(11): e61-e61.

Miyakawa, Isamu. 2017. "Organization and Dynamics of Yeast Mitochondrial Nucleoids.” Proceedings of the Japan Academy. Series B, Physical and Biological Sciences 93(5): 339-59.

Miyakawa, Isamu, Masahiro Miyamoto, Tsuneyoshi Kuroiwa, and Nobundo Sando. 2004. "DNA Content of Individual Mitochondrial Nucleoids Varies Depending on the Culture Conditions of the Yeast Saccharomyces Cerevisiae.” Cytologia 69(1): 101-7.

Osman, Christof et al. 2015. "Integrity of the Yeast Mitochondrial Genome, but Not Its Distribution and Inheritance, Relies on Mitochondrial Fission and Fusion." Proceedings of the National Academy of Sciences 112(9): E947-56.

Puddu, Fabio et al. 2019. "Genome Architecture and Stability in the Saccharomyces Cerevisiae Knockout Collection.” Nature 573(7774): 416-20.

Pyle, Angela et al. 2016. "Reduced Mitochondrial DNA Copy Number Is a Biomarker of Parkinson's Disease." Neurobiology of Aging 38: 216.e7-216.e10.

Sagan, Lynn. 1967. "On the Origin of Mitosing Cells.” Journal of Theoretical Biology 14(3): 225-IN6.

Simon, V. R., S. L. Karmon, and L. A. Pon. 1997. "Mitochondrial Inheritance: Cell Cycle and Actin Cable Dependence of Polarized Mitochondrial Movements in Saccharomyces Cerevisiae.” Cell Motility 37(3): 199-210.

Solieri, Lisa. 2010. "Mitochondrial Inheritance in Budding Yeasts: Towards an Integrated Understanding." Trends in Microbiology 18(11): 521-30.

Stevens, Barbara. 1981. "Mitochondrial Structure.” In The Molecular Biology of the Yeast Saccharomyces: Life Cycle and Inheritance, Cold Spring Harbor Laboratory Press, 471-504.

Wai, Timothy et al. 2010. "The Role of Mitochondrial DNA Copy Number in Mammalian Fertility1." Biology of Reproduction 83(1): 52-62.

Westermann, Benedikt. 2014. "Mitochondrial Inheritance in Yeast.” Biochimica et Biophysica Acta (BBA) - Bioenergetics 1837(7): 1039-46.

Williamson, D. H., and Ethel Moustacchi. 1971. "The Synthesis of Mitochondrial DNA during the Cell Cycle in the Yeast Saccharomyces Cerevisiae." Biochemical and Biophysical Research Communications 42(2): 195-201. 
Williamson, Donald H. 2002. "The Curious History of Yeast Mitochondrial DNA.” Nature Reviews Genetics 3(6): 475-81.

Williamson, Donald H., and P. P. Slonimski. 1976. "Packaging and Recombination of Mitochondrial DNA in Vegetatively Growing Yeast Cells." In Genetics, Biogenesis, and Bioenergetics of Mitochondria, Berlin: De Gruyter, 117-24.

https://www.degruyter.com/document/doi/10.1515/9783111522241-012/html (June 22, 2021).

Zeviani, Massimo, and Stefano Di Donato. 2004. "Mitochondrial Disorders.” Brain 127(10): 2153-72. 


\section{Methods}

\section{Strains}

yJHK112, a haploid, prototrophic, heterothallic, MATa, BUD4-corrected, and ymCherry-labeled W303 strain (Koschwanez et al., 2013) was used as the haploid lab strain in this paper. We created the diploid lab strain from this strain by transforming (Gietz and Schiestl 2007) the haploid lab strain with plasmid pRY003, temporarily providing a functional HO locus allowing mating type switching and subsequent mating. pRY003 was a gift from John McCusker (Addgene plasmid \#81043;

http://n2t.net/addgene:81043; RRID:Addgene_81043). The ploidy of the resulting strain was confirmed by (1) ability to produce tetrads after plating to sporulation media; (2) by flow cytometry for total genomic content; and (3) by the presence of a PCR product for both the MATa and MATa loci. YPS623, the wild diploid strain used in this paper, was isolated from Mettler's Woods, NJ as described in (Sniegowski et al., 2002). YPS 2066, the haploid wild strain used in this paper, originated from an ascospore of YPS623, isolated by microdissection, and subsequently made heterothallic by knocking out the $H O$ locus.

\section{Growth conditions}

All growth was carried out in liquid media in Erlenmeyer flasks kept at 30C and shaken at $200 \mathrm{rpm}$. An initial growth cycle was carried out in $6 \mathrm{~mL}$ media in a $50 \mathrm{~mL}$ flask. Unless otherwise noted, all experiments described in this paper were carried out in $50 \mathrm{~mL}$ media in a $250 \mathrm{~mL}$ flask. Except for the experiment described in Fig. 4, all transfers to start experiments were 1:2000 ( $25 \mathrm{ul}$ into $50 \mathrm{~mL}$ ) dilutions from pregrown cultures. Three kinds of media were used: YPD ( $2 \%$ peptone, $2 \%$ glucose, $1 \%$ yeast extract); YPG (2\% peptone, $3 \%$ glycerol, $1 \%$ yeast extract); and YPEG (2\% peptone, $2.6 \%$ glycerol, $2.6 \%$ ethanol, $1 \%$ yeast extract).

\section{qPCR}

qPCR assays for mtDNA content were performed using the relative standard curve method. For both lab and wild strains, a single large-scale extraction from the haploid strain was performed when the culture was in fermentation, at absorbance $\mathrm{A}_{600}=0.6$. For every $q \mathrm{PCR}$ run, a fivefold serial dilution of this extract served as the standard curve. Each sample was run with two sets of primers, identical to the primers employed in (Puddu et al., 2019). We employed one set of primers to target a region of genomic DNA and another to amplify a region of mitochondrial DNA. The gDNA primer set (fwd: TGCTTTGTCAAATGGATCATATGG , rev: CCTGGAACCAAGTGAACAGTACAA) targeted a short region of GAL1 (chr II: 280382-280459) and the mtDNA primer set (fwd: CACCACTAATTGAAAACCTGTCTG, rev: GATTTATCGTATGCTCATTTCCAA) targeted a short region of COX1 in the mtDNA (25574-25686). For each sample, the quantity of mtDNA and gDNA relative to the standard curve was computed, and the ratio of the two recorded as the relative mtDNA copy number. Threshold calculations, curve fitting, and quantity calculations were performed by the QuantStudio 3 software. All wells were run in triplicate per run and outliers from each triplet were occasionally removed manually. Each qPCR run was performed on a 96-well plate allowing a maximum of 11 unknown samples to be assayed for relative mtDNA copy number (11 samples $\mathrm{x} 2$ primer sets $\mathrm{x}$ in triplicate $=66$ wells, plus 5 standards $\mathrm{x} 2$ primer sets $\mathrm{x}$ in triplicate $=30$ wells, for a total of 96 wells). 


\section{DNA extractions}

DNA extractions were performed using Zymo Quick-DNA Fungal/Bacterial kits (D6005), which employ glass beads to physically lyse cells followed by a spin column-based purification. Because extractions were performed from growing cultures at different time points, within a single experiment the extraction volume was adjusted so that the absolute number of cells extracted was similar for each timepoint, in order to limit any effects of different extraction conditions. As an exception to this practice, extractions destined for whole-genome sequencing were larger in cell number than extractions destined for qPCR only.

Similar results as to those described here were obtained with both purely enzymatic extraction protocols (Zymo D2002) and a non-kit-based glass beads + phenol:chloroform protocol.

\section{Density measurements}

Density measurements were performed by manually counting the number of cells, via microscope, in an improved Neubauer-style hemocytometer. Depending on culture density, dilutions of up to 1/200 (in water) were performed before counting. Still-attached buds were counted as a separate cell if having a diameter greater than half that of the mother. Density was also tracked by measuring absorbance at 600 $\mathrm{nm}\left(\mathrm{A}_{600}\right)$, and referring to a standard curve created from many simultaneous measurements of absorbance and cell count.

\section{Sequencing and copy number analysis}

The mtDNA copy number analysis was conducted essentially following (Puddu et al., 2019) and was carried out working from short-read (Illumina) WGS data as follows. Reads were first processed by trim_galore with options --nextera --stringency 3 --paired --quality 20 --

length 50. Reads were mapped to the S288C R64 reference genome by bwa. Duplicate reads were removed by biobambam2's bammarkduplicates and then read depth computed by samtools depth. The mtDNA genome includes many regions of high AT content that are not covered well in WGS. Hence the mtDNA copy number was computed as the median read depth in a region of the mtDNA genome with regular coverage (COX1 from 14000-20000; see Fig. S3) divided by the median read depth in the nuclear genome. mtDNA copy number calculated using COX3 instead (79213-80022) was very similar (Fig S1). We validated our copy-number estimation procedure by calculating copy numbers for other repetitive elements (Fig S1).

For long-read (Nanopore) data, reads were mapped via minimap2, and then samtools depth used to obtain read depths as for the short-read data. mtDNA coverage for long-read data in shown in Fig. $\mathrm{S} 3$; it is somewhat more regular than for short-read sequencing. mtDNA copy number estimates from the two sequencing methods, performed on the same extract, were extremely similar (Table S2).

\section{Data and code}

[[Specify where data and code are hosted online.]] 


\section{Supplementary Figures and Tables}

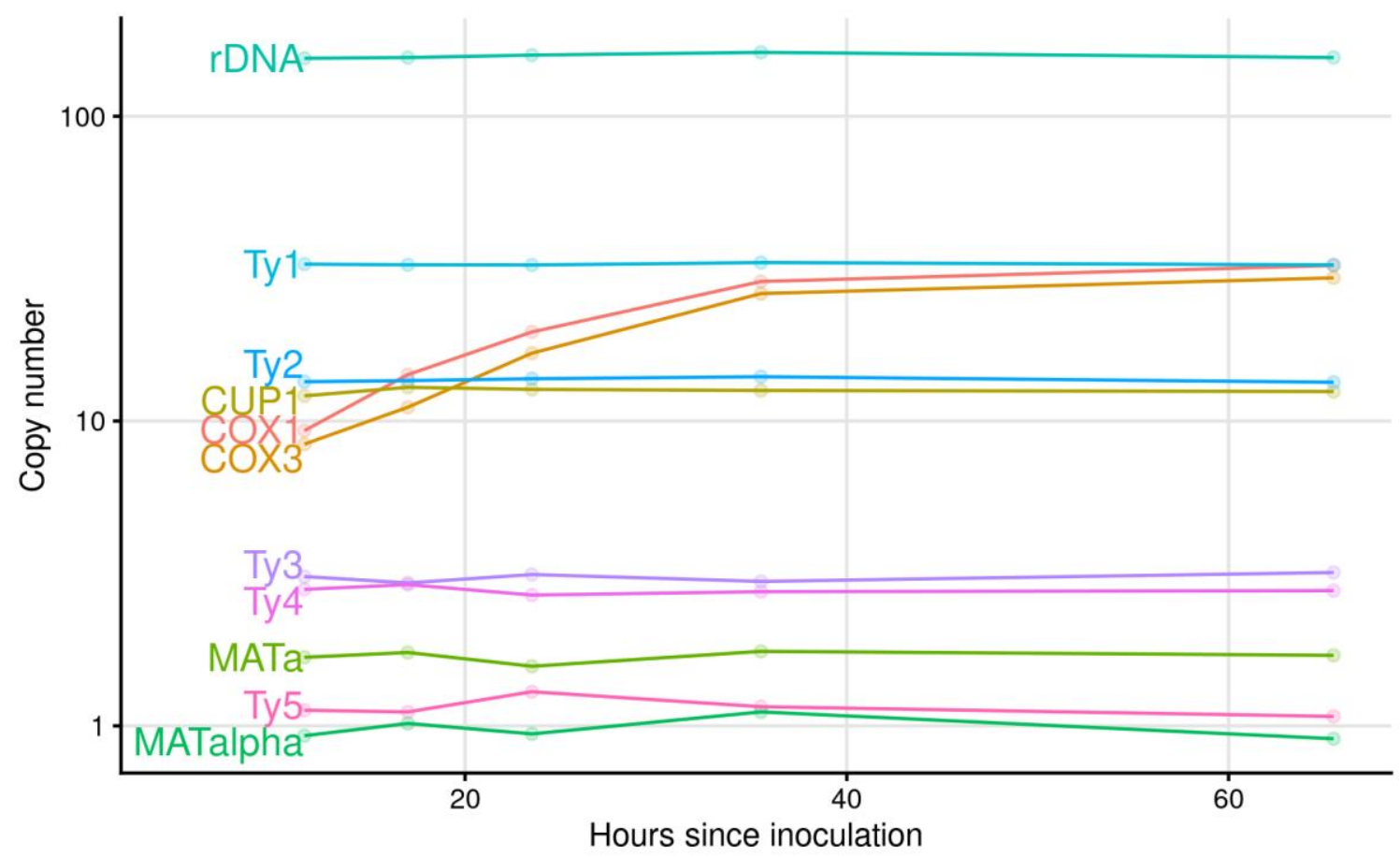

Fig. S1. The mitochondrial $C O X 1$ and $C O X 3$ genes increase in copy number over time. $C O X 1$ provides the data for Fig. 1C. As controls, we assayed the copy number of 9 gDNA loci: ribosomal DNA, CUP1, $M A T a, M A T \alpha$, and the 5 Ty transposons.

Table S2. Long-read sequencing gives near identical copy number results to short-read sequencing for identical extracts.

\begin{tabular}{|l|l|l|}
\hline & Illumina & Nanopore \\
\hline Fermentation & 8.6 & 8.2 \\
\hline Respiration & 27.8 & 28.5 \\
\hline
\end{tabular}




\section{Short-read mtDNA coverage}

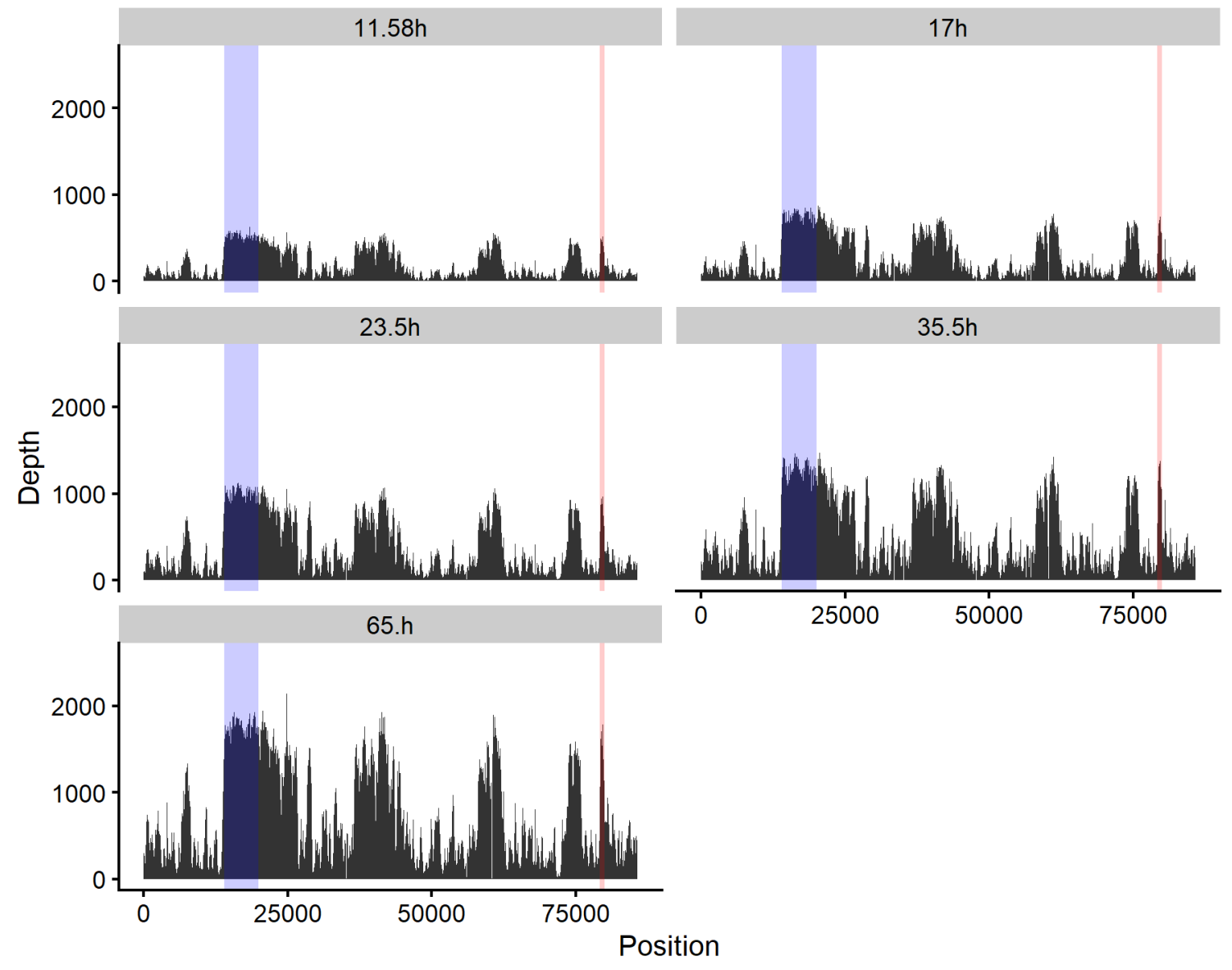

Fig S3. From short-read (Illumina) sequencing, mtDNA read depth from the 5 timepoints sequenced, with regions of mtDNA used for determining copy number highlighted (COX1 in blue; COX3 in red). 


\section{Long-read mtDNA coverage}

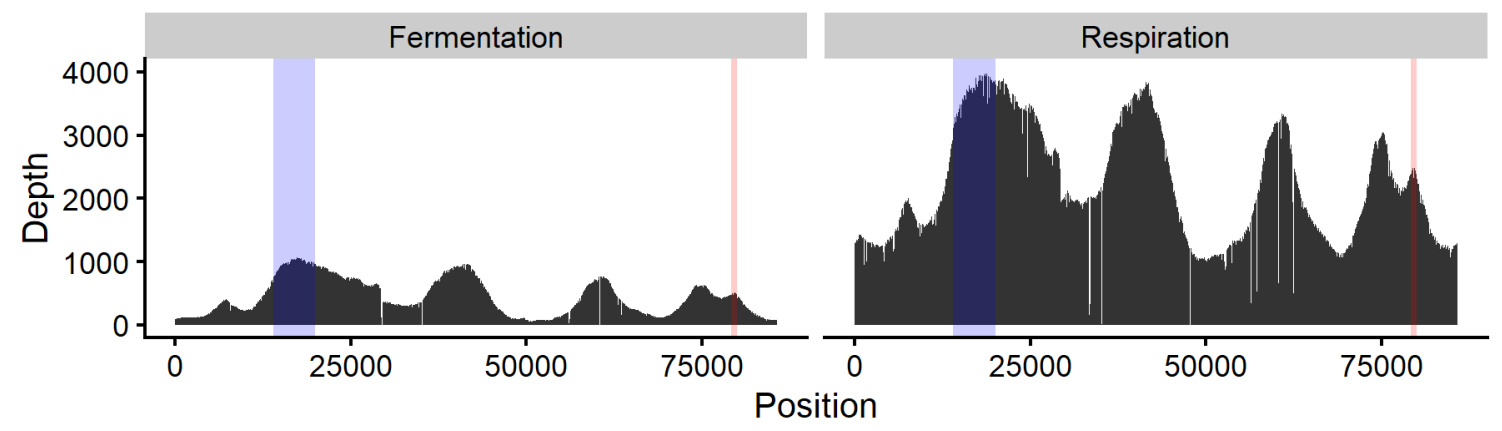

Fig S4. From long-read (Nanopore) sequencing, mtDNA read depth, with regions of mtDNA used for determining copy number highlighted (COX1 in blue; COX3 in red).
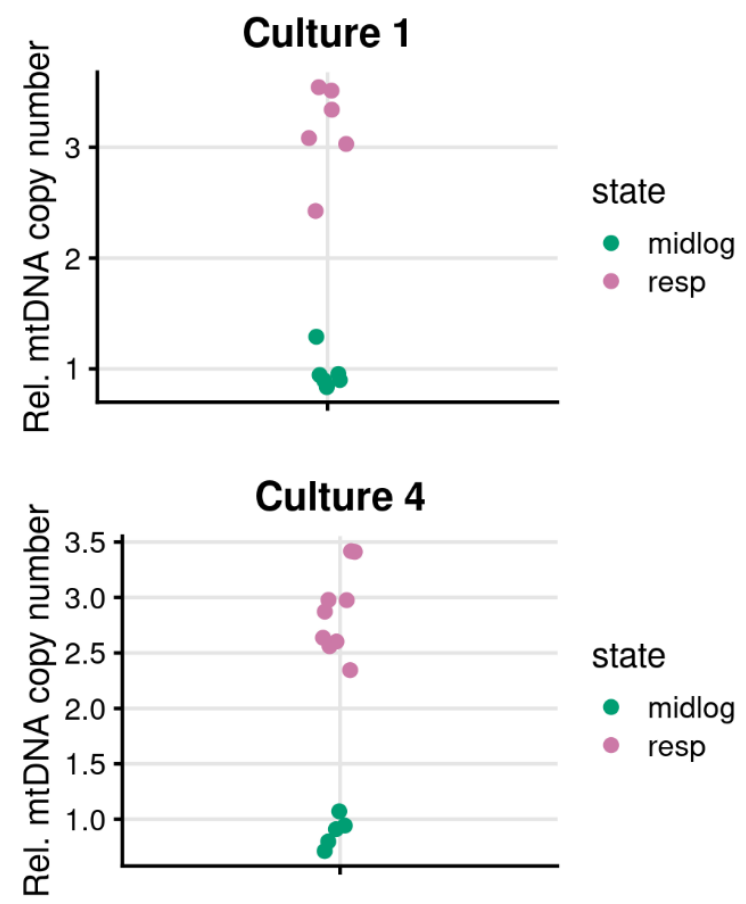

Fig S5. A culture of the haploid lab strain was inoculated as described in Methods, and then three consecutive serial transfers were performed during the fermentation phase, to create Cultures 1-4. Extracts were taken from both fermentation and respiration phase of Cultures 1 and 4 . This experiment shows that even after $\sim 60$ hours in fermentation, the mtDNA copy number remains low, ruling out the possibility that copy number increases solely as a function of time. 